\title{
REVISIÓN DE EVIDENCIAS \\ DE LAS TÉCNICAS DE DIR/FLOORTIMETM \\ PARA LA INTERVENCIÓN EN NIÑOS \\ Y NIÑAS CON TRASTORNOS \\ DEL ESPECTRO DEL AUTISMO
}

\section{Review of evidence of DIR/Flortime ${ }^{\mathrm{TM}}$ techniques for intervention in children with Autism Spectrum Disorders}

Liliana Rojas TORRes

Universitat de Valencia. Facultad de Psicología.

Departamento de Psicología Evolutiva y de la Educación

Yurena Alonso Esteban

Universitat de Valencia. Facultad de Psicología.

Departamento de Psicología Evolutiva y de la Educación

Francisco Alcantud-Marín

Universitat de Valencia. Facultad de Psicología.

Departamento de Psicología Evolutiva y de la Educación

Francisco.Alcantud@uv.es

Recepción: 17 de octubre de 2019

Aceptación definitiva: 3 de marzo de 2020

Resumen: Las técnicas de DIR/Floortime ${ }^{\mathrm{TM}}$ han tomado en nuestro país un cierto impulso. El objetivo de este artículo es analizar las evidencias sobre la eficacia que estas técnicas, para lo cual se han realizado búsquedas en cuatro bases de datos (PsychArticles, ERIC, PubMed y Medline). Después de depurar los documentos encontrados aplicando los criterios de inclusión/exclusión, se han detectado solo 18 estudios empíricos (7 estudios de caso único, 3 estudios cuasi-experimentales y 6 ensayos clínicos aleatorizados). Dos de los estudios son reanálisis de estudios anteriores o estudios de seguimiento. Una vez valorados los diferentes estudios, se concluye que es necesaria más investigación 
REVISIÓN DE EVIDENCIAS DE LAS TÉCNICAS DE DIR/FLOORTIMETM PARA LA INTERVENCIÓN EN NIÑOS Y NIÑAS CON TRASTORNOS DEL ESPECTRO DEL AUTISMO

LILIANA ROJAS TORRES, YURENA ALONSO ESTEBAN Y FRANCISCO ALCANTUD-MARÍN

sobre las técnicas de DIR/Floortime ${ }^{\mathrm{TM}}$. Aunque las evidencias apuntan hacia la eficacia de la incorporación de los padres y las técnicas de juego en el suelo, los estudios son muy heterogéneos y no permiten realizar ningún metaanálisis de resultados para evidenciar su eficacia.

Palabras clave: Trastorno del Espectro Autista; intervención temprana; interacción padres-hijos; DIR/Floortime ${ }^{\mathrm{TM}}$.

Aвstract: The techniques of DIR/Floortime ${ }^{\mathrm{TM}}$ have taken a certain momentum in our country. The aim of this article is to analyze the evidence on the efficacy of these techniques for which four databases have been searched (PsychArticles, ERIC, PubMed and Medline). After refining the documents found by applying the inclusion/exclusion criteria, only 18 empirical studies were detected (7 single case studies, 3 quasi-experimental studies and 6 randomized clinical trials). Two of the studies are re-analyses of previous studies or follow-up studies. Once the different studies have been evaluated, it is concluded that more research is needed on the techniques of DIR/Floortime ${ }^{\mathrm{TM}}$. Although the evidence points to the efficacy of parental involvement and soil play techniques, the studies are very heterogeneous and do not allow any meta-analysis of results to show their efficacy.

KeY words: Autism Spectrum Disorder; early intervention; parent-child interaction; DIR/Floortime ${ }^{\mathrm{TM}}$.

\section{Introducción}

L

os Trastornos del Espectro del Autismo (en adelante TEA) son trastornos del neurodesarrollo que se caracterizan por déficits persistentes en la comunicación e interacción social y patrones repetitivos y restringidos de conductas, actividades e intereses que causan alteraciones significativas en su desarrollo a nivel social, laboral o en otras áreas del funcionamiento. Los síntomas deben estar presentes en el periodo de desarrollo temprano (antes de los 36 meses de vida) (APA, 2013). De acuerdo con el Centro de Control de Enfermedades (CDC) ${ }^{1}$, este trastorno afecta a 1/59 niños y niñas en EE. UU. (Baio et al., 2018). En la actualidad, aún no se conoce una etiología única del trastorno, apuntando hacia varias causas posibles que pudieran estar en la base de los trastornos del neurodesarrollo desencadenantes.

Las evidencias apoyan que algunos programas o técnicas de intervención, aprovechando la plasticidad cerebral de los primeros años de vida (Artigas-Pallarés, 2007; Dawson et al., 2012), pueden llegar a mitigar las características sintomatológicas de los Trastornos del Espectro del Autismo, logrando un mayor desarrollo de la inteligencia, habilidades sociales, comunicación y lenguaje y mejorando su calidad de vida (Eldevik et al., 2009; Fein et al., 2013; Kasari, Gulsrud, Freeman, Paparella y Hellemann, 2012; Makrygiam y Reed, 2010; Reichow, Barton, Boyd y Hume, 2014;

1 CDC del inglés "Center for Disease Control".

Ediciones Universidad de Salamanca / CC BY-NC-ND

Siglo Cero, vol. 51 (2), 2020, abril-junio, pp. 7-32 
Warren et al., 2011). Existe un mayor reconocimiento de la eficacia de las técnicas y métodos basados en el desarrollo infantil y la aplicación de principios de análisis conductual (Simith y Iadarola, 2015), incluyendo ya programas más evolucionados en los que se enfatiza el uso de escenarios de aprendizaje estructurados, control de estímulo, desarrollo de rutinas, ambientes naturales, etc. (Lang, Hancock y Singh, 2016). Sin embargo, no todos los estudios incorporan diseños rigurosos desde el punto de vista metodológico. Las dudas sobre la eficacia de las técnicas generan una situación de incertidumbre entre los padres y cuidadores que incrementa la tensión en las familias. A menudo están ansiosos por inscribir a sus hijos en programas prometedores enfrentándose a los financiadores (Seguridad Social o compañías de seguros) que rechazan el pago de programas de intervención que consideran con escaso apoyo empírico (Rogers y Vismara, 2008). En otras ocasiones, optan por inscribir a sus hijos a varios programas de intervención. Lo que parece obvio es que se hace necesaria una continua revisión de las evidencias que aportan las diferentes técnicas o métodos en forma de guías de buenas prácticas (Fuentes-Biggi et al., 2006).

Sin duda, los programas de intervención basados en principios operantes (ABA) son los que han desarrollado mayor número de estudios que evidencian su eficacia (Eldevik et al., 2009; Makrygianni y Reed, 2010; Reichow, 2012). Sin embargo, las críticas se han centrado fundamentalmente en la falta de generalización de los resultados, desde ambientes clínicos controlados, hacia ambientes más naturales o la aparición de conductas disruptivas o de huida. Con el tiempo, se han desarrollado otros enfoques terapéuticos que, sin olvidar los principios operantes, se centran en el desarrollo del niño. Este segundo bloque de técnicas de intervención pueden clasificarse bajo el epígrafe de Intervenciones en el Desarrollo Social Pragmático (DSP)², su característica general es que buscan desarrollar habilidades funcionales en una secuencia consistente con el desarrollo típico infantil, centrándose en las capacidades relacionadas con la comunicación en contextos sociales apropiados más que en el comportamiento concreto (Prizant y Wetherby, 1998). El método centrado en el desarrollo más conocido es el "Early Start Denver Model” (ESDM); en este programa los padres reciben entrenamiento por parte de los profesionales para que sean capaces de replicar lo aprendido en terapia en otros contextos del niño. El objetivo principal es lograr avances en el desarrollo funcional, las habilidades sociales y el desarrollo del lenguaje, así como incrementar la atención, motivación y mejorar las dinámicas familiares (Rogers et al., 2012; Rogers et al., 2014; Vivanti et al., 2014; Rogers et al., 2018).

Se han desarrollado también técnicas específicas para mejorar la calidad y la cantidad de interacciones entre padres e hijos incorporando a los padres como agentes activos en la intervención. El objetivo de la incorporación de los padres es múltiple, por una parte, hacer que los programas de intervención intensiva sean más accesibles en términos económicos y geográficos y, por otra, a nivel terapéutico, conseguir mejorar la calidad de las interacciones padres-hijos durante el juego en vivo, primero en la clínica bajo la tutela de los profesionales para, después, saltar a entornos naturales y de forma autónoma. Por ejemplo, la Terapia de interacción padres-hijos (The

2 DSP del ingles Developmental Social Pragmatic.

Ediciones Universidad de Salamanca / CC BY-NC-ND

Siglo Cero, vol. 51 (2), 2020, abril-junio, pp. 7-32 
Parent-Child Interaction Therapy [PCIT]; Eyberg, 1988) tenía como objetivo reducir la aparición de comportamientos desafiantes y disruptivos, fomentar la imitación verbal, mejorar el lenguaje y la comunicación social y emocional a través de técnicas tradicionales de terapia de juego infantil.

Desde hace ya tiempo se conoce el valor del juego en el desarrollo psicológico del niño (Piaget, 1952). Su aplicación a nivel terapéutico también es reconocida desde ya hace tiempo (Ginott, 1959; Ginott, 1960). El juego es una actividad universal en todos los niños mediante la cual ensayan situaciones problema, por lo que podemos considerarlo como la clave para desarrollar conductas sociales (Mastrangelo, 2009). Las técnicas de intervención centradas en el juego con niños con TEA se han utilizado desde ya hace tiempo, incluso se ha evidenciado su eficacia en estudios de metaanálisis (Leblanc y Ritchie, 2001; Bratton, Ray, Rhine y Jones, 2005; Hillman, 2018), con resultados que demuestran los cambios producidos en el desarrollo socioemocional y comunicativo.

Algunos modelos intentan integrar varias de estas ideas como es el modelo DIRC (Developmental, Individual Difference, Relationship-Based) (Greenspan y Wieder, 1997a; Greenspan y Wieder, 2006; Wieder y Greenspan, 2003). DIR@ es un marco teórico interdisciplinar para el diseño de intervenciones globales que permita el desarrollo funcional del niño o niña, basándose en su perfil individual, sus relaciones familiares y los patrones de interacción (Greenspan y Wieder, 1999). Integra, por una parte, la investigación en el desarrollo psicológico del niño en general (Garner, Jones y Miner, 1994) y del niño con necesidades específicas en particular (Greenspan y Wieder, 1998), reconociendo la importancia de las interrelaciones tempranas y el buen funcionamiento familiar en el desarrollo del niño (Greenspan, 1992). El objetivo del modelo es potenciar una comunicación espontánea entre el niño y sus cuidadores, haciendo crecer sus interrelaciones y promoviendo un compromiso alegre y placentero para ambos que haga incrementar el número y la calidad de las interacciones.

La forma práctica de la intervención es el tiempo de juego en el suelo (FloorTime Play-FTP). Consiste en desarrollar o alentar sesiones de juego espontáneas y estructuradas o no, en las que se construyen relaciones y se desarrollan la autorregulación, la comunicación bidireccional, el compromiso social, el pensamiento complejo y la resolución de problemas (Wieder y Greenspan, 2003). Las grandes diferencias entre los perfiles de los niños con TEA obligan a un enfoque de intervención individual teniendo en cuenta también su familia y entorno de desarrollo. El objetivo del programa individual será incrementar las competencias en los déficits centrales que presente el niño o niña contribuyendo a construir las bases para que pueda relacionarse, comunicarse y pensar por sí mismo.

Una sesión de FTP (Greenspan y Wieder, 1998) se desarrolla en un entorno lo más natural posible para el niño y requiere que el terapeuta o el padre se sienten en el suelo al nivel del niño y jueguen con él. El propósito es ayudar al niño a alcanzar los hitos evolutivos no desarrollados en su momento, volviendo sobre ellos. Durante cada sesión, el padre o el terapeuta siguen el ejemplo del niño. Esta imitación y respeto ayuda a establecer una relación entre el niño y el adulto a partir de la cual se desarrollarán las habilidades sociales, emocionales y de comunicación básicas. 
Durante una sesión de FTP, el niño aprende a relacionarse, a iniciar acciones, a conocer sus propios deseos y a darse cuenta de que sus acciones pueden provocar respuestas de los demás. Las actividades desarrolladas en las sesiones de FTP se centran en los intereses del niño y, en consecuencia, son motivadoras para él dado que es quien las ha elegido. Además, el entorno natural y conocido de cada sesión contribuye a calmarlo y mejorar el nivel de confort.

DIR/Floortime ${ }^{\mathrm{TM}}$ es un método de intervención patentado por ICDL (Interdisciplinary Council on Developmental and Learning Disorders), organización sin ánimo de lucro que tiene como misión, entre otras, la formación y acreditación en el uso del modelo de intervención. Dispone de un manual de intervención (Greenspan y Wieder, 2006). Sin embargo, las evidencias y estudios sobre los resultados del DIR/ Floortime $^{\mathrm{TM}}$ son escasos (IECS, 2007; Case-Smith y Arbesman, 2008), al margen de los realizados por los autores del método. En este artículo pretendemos realizar una búsqueda sistemática para determinar las evidencias de su efectividad.

\section{Método}

Con la finalidad de localizar el máximo número de documentos relacionados con la intervención con el modelo DIR/Floortime ${ }^{\mathrm{TM}}$, desarrollamos una revisión sistemática siguiendo los criterios de búsqueda, selección y evaluación de los documentos recomendada por el Centro de Revisión y Difusión del NHS (CRD, 2008) y las directrices del grupo PRISMA (Preferred Reporting Items for Systematic Reviews and Meta-Analyses Guidelines) (Moher, Liberati, Tetzlaff y Altman, 2009).

Se realizaron búsquedas en cuatro bases de datos (ProQuest-PsychArticles, ProQuest-ERIC, ProQuest-PubMed y Web of Science-Medline). Dada la cantidad de publicaciones y artículos, solo se seleccionaron los publicados en revistas científicas con una revisión por pares doble ciego. La búsqueda se realizó accediendo a todas las bases de datos utilizando la interfaz de búsqueda en línea TROBES del Servicio de Documentación y Biblioteca de la Universidad de Valencia (España).

\subsection{Estrategia de búsqueda}

Los términos de búsqueda utilizados fueron (“Autism” OR "Pervasive Developemntal Disorders”) AND (“DIR Model” OR “Floortime”). Estos términos pueden aparecer en cualquier parte del documento indexado. La misma estrategia se adaptó a todas las bases de datos buscadas.

\subsection{Criterios de inclusión}

La selección ha sido delimitada al periodo comprendido entre los años 1990-2018. Se incluyeron solo los artículos que aportaran datos empíricos de los resultados de la 
intervención. Sobre los artículos seleccionados, se analizó la bibliografía utilizando la llamada técnica de la bola de nieve, con el fin de detectar algún otro estudio no indexado en las bases de datos.

\subsection{Criterio de exclusión}

Los artículos encontrados siguiendo los criterios de la búsqueda fueron 123 de los cuales se encontraron una totalidad de 32 artículos duplicados. Posteriormente se realizó la lectura de los resúmenes de los artículos y se encontraron 74 artículos en los cuales se exponía el modelo DIR desde un punto de vista teórico o artículos considerados de divulgación y difusión del modelo o solo se nombra el modelo DIR/Floorti$\mathrm{me}^{\mathrm{TM}}$ como encuadre teórico y comparativo sobre el modelo objeto de estudio.

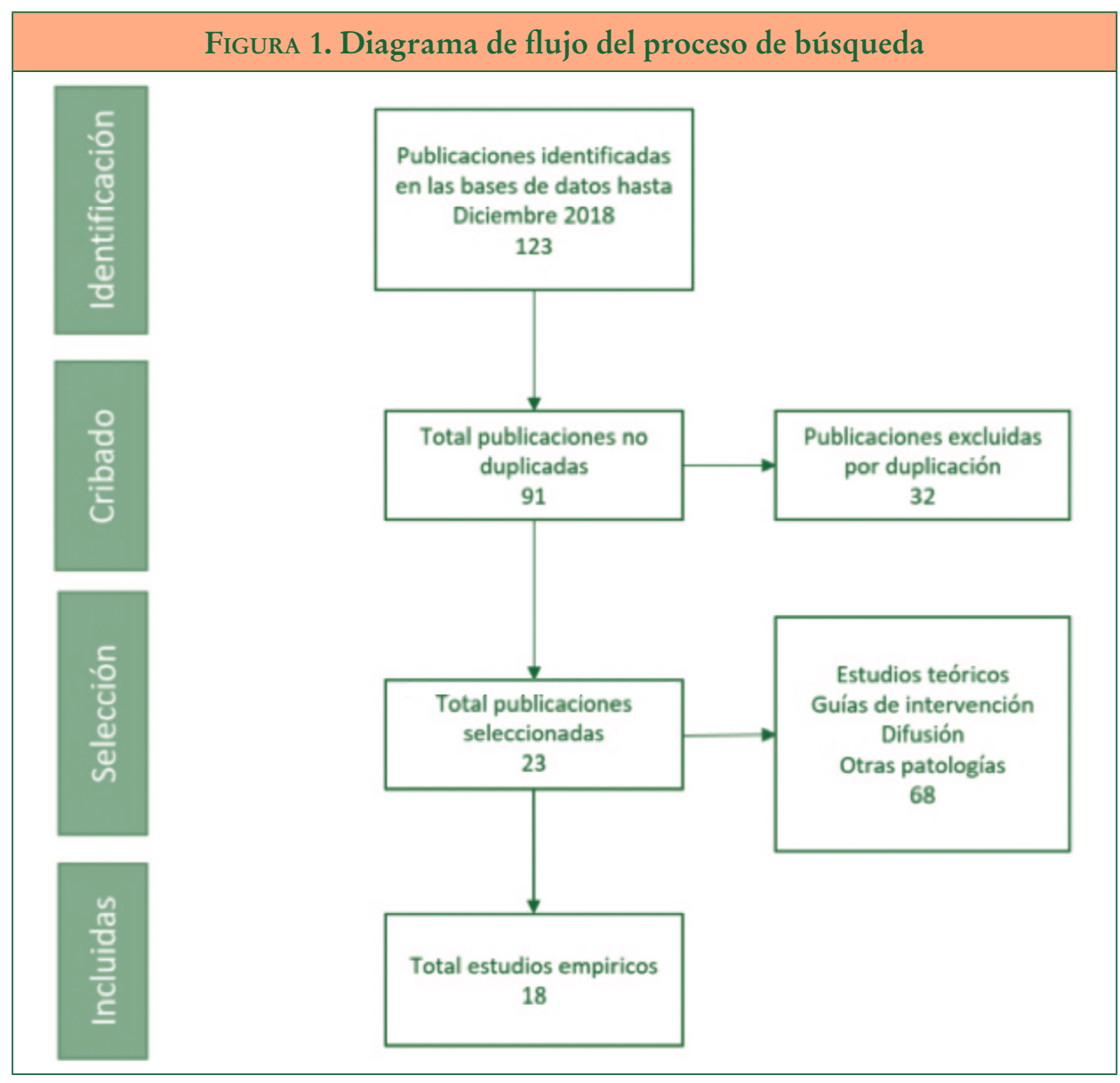

Ediciones Universidad de Salamanca / CC BY-NC-ND

Siglo Cero, vol. 51 (2), 2020, abril-junio, pp. 7-32 
REVISIÓN DE EVIDENCIAS DE LAS TÉCNICAS DE DIR/FLOORTIMETM PARA LA INTERVENCIÓN EN NIÑOS Y NIÑAS CON TRASTORNOS DEL ESPECTRO DEL AUTISMO

LILIANA ROJAS TORRES, YURENA ALONSO ESTEBAN Y FRANCISCO ALCANTUD-MARÍN

\section{Resultados}

Se han encontrado un total de 18 estudios que aportan resultados empíricos en la aplicación del modelo de intervención DIR/Floortime ${ }^{\mathrm{TM}}$. Seis de ellos son estudios de casos, cuatro son ensayos clínicos con pretest y postest, seis con ensayos clínicos aleatorizados y dos son estudios de seguimiento de estudios previos.

\subsection{Estudios de casos}

En nuestra búsqueda hemos encontrado seis estudios que pueden clasificarse como de caso único, aunque con diferencias entre ellos. El estudio más significativo es el desarrollado por Greenspan y Wieder (1997b), que examinaron los registros clínicos de la consulta clínica privada de más de 200 niños durante un periodo de 8 años. Todos los niños accedieron a la consulta por problemas de comunicación y relación y fueron diagnosticados con Trastornos Generalizados del Desarrollo No Específico o Autismo según criterios del DSM IV (APA, 1994). Todos los niños tenían entre los 22 meses y los 4 años de edad, con diferentes niveles de gravedad según puntuación en el CARS (Childhood Autism Rating Scale: Schopler, Reichler y Renner, 1986) y habían sido tratados bajo el enfoque DIR/Floortime ${ }^{\mathrm{TM}}$ al menos durante dos años. El desarrollo del funcionamiento emocional fue evaluado mediante la escala FEAS (Funtional Emotional Assessment Scale: Greenspan, 1992; Greenspan y DeGangi, 2001) aplicada después de cada consulta de seguimiento. Los resultados ofrecidos, aunque con grandes limitaciones de orden metodológico (se trata de un diseño ex-post-facto con ausencia de grupo de control, limitaciones en la selección de los casos, falta de representatividad de la diversidad sintomatológica, mayor implicación de las familias, etc.), apuntan hacia cambios cualitativos producidos por la intervención. Así, el $58 \%$ de los niños tuvieron mejoras buenas o excepcionales en habilidades sociales, tareas cognitivas y habilidades académicas el $25 \%$ de los niños solo mejoraron en términos medios, y el $17 \%$ restante continuaron con dificultades comunicativas y de relaciones. Estas mejorías se combinaron con una disminución significativa de conductas de ensimismamiento, conductas de evitación o escape, aislamiento y autoestimulación.

Los mismos autores (Wieder y Greenspan, 2005) realizan un seguimiento entre diez y quince años a 16 niños y sus familias, participantes del estudio anterior y sobre los que siguieron desarrollando sesiones de DIRC. El desarrollo emocional de estos niños/adolescentes demuestra que, en algunos casos, niños previamente diagnosticados como TEA pueden llegar a desarrollar habilidades académicas y un pensamiento empático, creativo y reflexivo con relaciones sociales con sus iguales satisfactorias. Es interesante la aportación de las características de este grupo de éxito, todos ellos iniciaron la intervención entre los 12 y 24 meses y fueron diagnosticados entre los 24 y 30 meses de edad. Este estudio aporta descripciones sobre la evolución del proceso, pero adolece de cuantificación métrica del mismo por lo que resulta imposible atribuir las mejoras al uso del método DIR/Floortime ${ }^{\mathrm{TM}}$.

Los estudios de caso único mejor descritos son los de Hilton y Seal (2007) y Dionne y Martini (2011). En el primero de ellos (Hilton y Seal, 2007), se realiza un 
estudio de caso único con dos hermanos gemelos monocigóticos, desarrollando un programa de intervención de 18 sesiones con los modelos DIR@ y ABA (Lovaas, 1987). El objetivo fue determinar cuál de las metodologías tiene mayor eficacia y ayudar a los padres a la elección del programa más adecuado para sus hijos. La intervención fue realizada por los profesionales durante un periodo de 9 semanas, aunque las actividades eran similares para los dos niños la implementación varió según el tipo de intervención. En el caso de ABA, se realizaron sesiones más estructuradas en donde primó el uso de reforzadores tangibles y en el caso de DIR@, se utilizó la motivación del niño para la elección y realización de las actividades. Los resultados se contrastaron utilizando como pretest y postest el CSBS (Communication and Symbolic Behavior Scales: Wetherby y Prizant, 1999), obteniendo resultados contradictorios. Mientras que en reciprocidad y conducta simbólica se observaron incrementos en el niño intervenido según el modelo DIR@, en la dimensión socioafectiva fue al contrario. A pesar de aportar una puntuación psicométrica, al tratarse de casos únicos no es posible aplicar ningún estadístico que determine la significación de las diferencias, por otra parte, en el artículo referenciado (Hilton y Seal, 2007) no se enumera ninguna medida preventiva para evitar el efecto cruzado entre los dos métodos fuera de la situación clínica.

Dionne y Martini (2011) presentan los resultados de una investigación de caso único con un niño de 3 años y 6 meses diagnosticado con autismo a los 2 años y 5 meses, para determinar la efectividad de la intervención Floortime ${ }^{\mathrm{TM}}$. Se realizaron cuatro sesiones durante 7 semanas con una duración de 45 minutos por sesión, todas las intervenciones fueron dirigidas de manera conjunta por los terapeutas y la madre del niño. Adicionalmente el niño estaba inscrito en un programa comunitario en centro infantil con apoyo de educador, terapeuta ocupacional y logopeda. Para evaluar el caso, se aplicaron las pruebas CARS, The Sensory Profile (Dunn, 1999) y FEAS a nivel descriptivo. Para evaluar la intervención se realizaron medidas observacionales específicas contabilizando el número de círculos de comunicación (CoC: Circles of Communication). Un CoC se refiere a un acto de comunicación recíproca entre dos participantes (niño y terapeuta o padres) que se responden entre ellos utilizando código verbal o no verbal. En total se desarrollaron 28 sesiones a lo largo de siete semanas de intervención. El análisis del efecto de la intervención solo se realizó sobre el número y calidad de CoC, para ello se analizaron las grabaciones en video de todas las sesiones que fueron codificadas por dos terapeutas de forma independiente. Los resultados evidenciaron mejoras en esta variable y los autoinformes realizados por la madre evidenciaron mejoras en la comunicación espontánea, relaciones familiares e intercambios durante la comunicación.

El resto de los estudios encontrados son de carácter descriptivo y cualitativo. Christian (2011) presenta un estudio cualitativo de dos casos de adultos. También hemos encontramos un estudio desarrollado en Taiwán con dos niños sin discapacidad intelectual (Yen, Tseng y Soong, 2008). En ambos casos solo presentan descripciones cualitativas. Lo mismo pasa con el estudio de Wieder y Greenspan (2003), que utilizan un caso como guion y demostración de los pasos a seguir en la intervención. Los resultados que aportan son descriptivos y cualitativos con un seguimiento de tres 
REVISIÓN DE EVIDENCIAS DE LAS TÉCNICAS DE DIR/FLOORTIMETM PARA LA INTERVENCIÓN EN NIÑOS Y NIÑAS CON TRASTORNOS DEL ESPECTRO DEL AUTISMO

LILIANA ROJAS TORRES, YURENA ALONSO ESTEBAN Y FRANCISCO ALCANTUD-MARÍN

años. Aunque los resultados manifiestan cambios cualitativos, la falta de evaluaciones métricas relativizan la importancia de estos cambios.

\subsection{Estudios cuasi-experimentales pre-postest}

Liao y colaboradores (2014) desarrollan en Taiwán un programa de intervención domiciliaria basado en los principios DIR/Floortime con el objetivo de mejorar la interacción social y el funcionamiento adaptativo. Los participantes fueron 11 niños con diagnóstico de TEA, la intervención tuvo una duración de 10 semanas (109,7 horas de intervención como promedio). Para obtener los resultados de la intervención se realizaron evaluaciones pre- y postintervención utilizando los siguientes instrumentos: FEAS (Greenspan y DeGangi, 2001), Vineland Adaptive Behavior Scales VABS-II (Sparrow, Cicchetti y Balla, 2005) y Parenting Stress Index-Short Form (PSI/SF: Abidin, 1990). El programa incluyó 3 semanas de entrenamiento a las madres en sesiones individuales en las que se desarrollaron objetivos individuales para cada niño. Al finalizar la intervención, realizada por las madres, se obtuvieron ganancias significativas en los seis dominios medidos por FEAS. En cuanto a la prueba VABS-II se demostraron incrementos significativos en el puntaje total y en los dominios de comunicación, habilidades de la vida cotidiana y habilidades sociales, sin embargo, el PSI no mostró cambios significativos en la percepción del estrés, pero las madres registraron mejoras en las interacciones con sus hijos. Aunque los resultados fueron positivos, este estudio no contó con un grupo control, lo que impide la generalización de los resultados obtenidos, así mismo el hecho de que las familias hayan participado de manera activa en otras intervenciones hace que los resultados y las puntaciones de las escalas puedan estar influenciados por otros factores.

En la misma dirección, Solomon, Nechleles y Ferch y Bruckam (2007) desarrollan un programa para entrenar a los padres en el juego con sus hijos con TEA basados en los principios de DIR/Floortime. El Proyecto PLAY (Play and Language for Autistic Youngsters) está diseñado para fortalecer el desarrollo funcional y disminuir la sintomatología autista y se enmarca en un programa de ámbito estatal PPHC (Play Project Home Consultation). En este estudio se quiso determinar la eficacia de la metodología en las habilidades parentales y las habilidades sociales de los niños. En la intervención participaron 68 niños diagnosticados con autismo y TGD, los padres recibieron entrenamiento intensivo del modelo DIR/Floortime y participaron de forma activa durante toda la intervención. El periodo de intervención fue de 14 meses (seis familias fueron excluidas al final por la falta de adherencia). Recibieron una visita domiciliaria al mes donde recibían instrucciones adicionales, modelado, entrenamiento, evaluación sobre sesiones grabadas en video y objetivos escritos para ayudar a implementar y ajustar la secuencia de habilidades. Todos los niños que participaron en esta investigación fueron evaluados al principio y al final utilizando la prueba FEAS. Los resultados obtenidos indican que no se presentaron cambios en el comportamiento de los cuidadores a partir de la intervención, sin embargo, se evidenció incremento en el desarrollo funcional; de igual manera, la mayoría de los padres 
reportaron mejoras en las relaciones, logrando intercambios en las interacciones de manera recíproca. Aunque este estudio expone información valiosa, presenta algunas limitaciones, una de ellas fue no incluir grupo control, lo cual hace imposible determinar que los resultados se atribuyen a la intervención PLAY Project, puesto que la mayoría de los niños participaban en otros programas de educación.

Aali et al. (2015) presentan una experiencia realizada en Mashhad (Irán) en la que diseñan una intervención centrada en la familia en combinación con DIR/Floortime. En total participaron 12 niños de 2 a 8 años y sus familias durante 5 meses de intervención. Aunque en el estudio se habla de tres grupos como grupos independientes (Terapia centrada en la familia, Floortime y grupo control), no se ha encontrado información detallada de la composición de estos ni del mecanismo de asignación a cada uno de ellos. Aunque se han utilizado escalas como la FEAS, se limitan a hacer valoraciones cualitativas.

\subsection{Ensayos clínicos aleatorizados}

Lal y Chhabria (2013) realizaron un estudio experimental en Mumbai (India) con pretest y postest y grupo control. Se seleccionaron 26 niños preescolares diagnosticados con autismo con edades comprendidas entre los 3 y los 6 años de cinco centros preescolares y consultas privadas de la localidad. Después de la selección fueron asignados a dos grupos de forma aleatoria quedando constituidos cada uno de ellos por 13 niños. Todos los niños fueron evaluados con la escala BSFS (Behavioral Scale for Social Skills: Lal y Chhanbria, 2013), desarrollada ad hoc para esta investigación. Al grupo experimental se aplicaron 20 sesiones de 30 minutos de Floortime, mientras que el grupo control siguió con su programa de intervención temprana habitual. Los resultados obtenidos demuestran en términos medios cambios significativos en todos los dominios evaluados con el BSFS entre pretest y postest en el grupo experimental y de magnitud superior a los encontrados en el grupo control. No obstante, los análisis estadísticos utilizados son débiles y no determinan la inexistencia de otras variables que pudieran explicar el efecto del tratamiento. Así mismo, no se aporta información psicométrica sobre la BSFS por lo que la validez de la medida puede ser cuestionada. Por otra parte, el proceso de selección y asignación aleatoria no está debidamente descrito y puede existir una correlación entre las puntuaciones del pretest y el postest. Por último, la intervención no cumple con todos los principios de la metodología DIR/Floortime ${ }^{\mathrm{TM}}$ y no se describe la participación de los padres.

Pajareya y Nopmaneejumruslers (2011) desarrollan en Tailandia un ensayo piloto controlado aleatorio con el fin de determinar los posibles beneficios adicionales que este método podría aportar a la intervención rutinaria. Se organizaron dos grupos, el primero solo recibió tratamiento convencional mientras que el segundo grupo recibió de forma suplementaria sesiones DIR/Floortime ${ }^{\mathrm{TM}}$. Los treinta y dos participantes fueron asignados a cada grupo utilizando una asignación aleatoria estratificada según edad y gravedad de los síntomas. Dado que resulta difícil o imposible poder controlar el efecto de la cointervención, se informó de las actividades realizadas al 
margen del programa de intervención. Los resultados evaluados con el CARS reflejan una mejora general en la gravedad de los síntomas TEA para todos los niños en ambos grupos, siendo esta más significativa en el grupo de intervención DIR/Floortime ${ }^{\mathrm{TM}}$. De la misma forma se utilizó una versión tailandesa de FEDQ (Functional Emotional Developmental Questionnaire: Greenspan y Greenspan, 2002) para valorar el desarrollo emocional del niño observando que el grupo de intervención obtuvo en el postest mejores resultados que el grupo control. Este estudio plantea algunos problemas metodológicos, el principal que los materiales y test debieron ser traducidos y se determinaron algunas diferencias culturales que pueden tener efecto en los resultados. En segundo lugar, que las familias participantes fueron voluntarias por lo que puede existir algún efecto de motivación adicional. Por último, los resultados ofrecidos no están debidamente segmentados haciendo referencia únicamente a los puntajes totales obtenidos en la aplicación de las escalas, sin explicar a profundidad las ganancias que se obtuvieron en cada uno de los dominios. Estos mismos autores realizan un segundo estudio (Pajareya y Nopmaneejumruslers, 2012), basado en los resultados de los participantes del grupo de intervención del estudio anterior, en donde realizaron un estudio de seguimiento para demostrar el efecto del mantenimiento y la adherencia al tratamiento de los padres, durante un año bajo el modelo de atención domiciliaria (Home-Based DIR/Floortime ${ }^{\mathrm{TM}}$ ). Los resultados apuntan en la misma dirección del estudio original, además de haberse logrado mejoras en las puntuaciones de cada una de las escalas en el contraste pretest postest, los padres continuaron relacionándose de manera positiva con sus hijos, lo cual generó mejorar en las relaciones familiares. En general, en los dos estudios se evidencia que los niños que más evolucionan son aquellos con síntomas menos graves. Esta evolución positiva está también determinada en cierta medida por el tiempo libre que los padres pueden pasar con sus hijos para ayudarles en su desarrollo. En este segundo estudio no se definió grupo control por lo que resulta imposible determinar si los resultados son atribuibles directamente a la intervención domiciliaria. Por otra parte, los resultados pueden estar contaminados por cointervención, dado que todas las familias acudieron a servicios comunitarios donde se aplicaban técnicas conductuales convencionales. Lo que sí se evidencia es que una intervención domiciliaria puede ser efectiva y complementaria a otros modelos de intervención.

Sealy y Glovinsky (2016) desarrollan un ensayo clínico controlado en Barbados con cuarenta díadas (padres-niño). Todos los niños tenían trastornos del neurodesarrollo y edades entre los 2 y los 7 años. Todos los niños presentaron trastornos del neurodesarrollo relacionados con la comunicación y la relación incluido TEA. Como referencia se utilizó el PDM (Psychodynamic Diagnostic Manual: PDM Task Force, 2006). Los niños fueron reclutados de centros de salud públicos y privados y asignados en dos grupos, el grupo experimental recibió intervención basada en DIR/ Floortime $^{\mathrm{TM}}$ y el grupo control (lista de espera) recibió tratamiento psicoeducativo convencional. En total participaron 40 díadas teniendo una duración de 12 semanas. El objetivo fue evaluar los cambios en el desarrollo del funcionamiento de la parentalidad reflectiva evaluada por el PDI (Parent Development Interview: Aber, Slade, Berger, Bresgi y Kaplan, 1985). El funcionamiento reflectivo de los padres es una 
capacidad interpersonal e intrapersonal que subyace en cómo los padres se sintonizan con el estado afectivo de sus hijos y cómo interpretan y responden a la experiencia subjetiva de sus hijos. El entrenamiento de los padres en DIR/Floortime ${ }^{\mathrm{TM}}$ mejoró su capacidad de funcionamiento reflectivo lo que sugiere que aprendieron a leer las demandas sociales de sus hijos y responder en consonancia. No obstante los resultados positivos, debemos hacer ver que el grupo no estaba formado exclusivamente por niños o niñas con TEA por lo que la comparación con otros estudios resulta compleja, también es posible que la heterogeneidad diagnóstica y las diferencias en la gravedad de los síntomas puedan estar afectando los resultados.

Solomon y colaboradores (Solomon, Van Egeren, Mahoney, Huber y Zimmerman, 2014) evaluaron el programa PPHC (Solomon, Necheles, Ferch y Bruckman, 2007), descrito en el apartado anterior, para lo que desarrollaron un ensayo controlado sobre un total de 128 familias con un hijo con diagnóstico de autismo. Se asignaron a dos grupos de 64 familias, de forma aleatoria, estratificando según edad y nivel de gravedad. El grupo control siguió recibiendo tratamiento estándar en los servicios comunitarios mientras que el grupo experimental recibió formación PLAY (el nivel de retención en el programa llegó al 89 \%). Los padres recibieron formación de DIR (C) y a lo largo de la intervención PLAY se realizaron visitas domiciliarias de 3 horas al mes durante doce meses. En estas visitas los padres recibieron entrenamiento por medio de videos, modelado, coaching, etc., enseñándoles a identificar las señales comunicativas sutiles y más difíciles de detectar y cómo responder contingentemente para mejorar la interacción. Las evaluaciones se hicieron por personal independiente sin conocimiento del estatus del niño (grupo control o experimental).

Para la obtención de los resultados se obtuvieron puntajes pretest y postest con las siguientes pruebas: ADOS (Lord, Rutter y Le Couteur, 1995), SCQ (Rutter, Bailey y Lord, 2003), FEAS, PSI y otras escalas. A partir de los datos obtenidos con la escala ADOS, los dos grupos demostraron mejoras en el diagnóstico, pero el grupo que recibió la intervención mostró mayores mejoras y estas fueron estadísticamente significativas. Con las otras pruebas se realizaron correlaciones con los resultados para determinar cambios en las interacciones padres e hijos, desarrollo funcional, estrés y depresión en los padres. En la interacción padre e hijos los resultados indican que los padres del programa experimental evidenciaron un cambio significativamente mayor en la calidad de las interacciones; en cuanto al desarrollo funcional el grupo experimental demostró cambios mayores que el grupo control, $y$, finalmente, los resultados de los padres no mostraron diferencias en los niveles de estrés entre los dos grupos. No obstante, el nivel de depresión de los padres del grupo experimental disminuyó en comparación con el grupo control. Esta investigación es una de las más potentes metodológicamente, sin embargo, una limitación de este estudio es que las horas dirigidas a la intervención y la interacción con los adultos variaron en los dos grupos, siendo el grupo control quien recibió menos horas y menos interacciones, lo cual puede tener influencia en los resultados obtenidos. Estos datos fueron revisados con posterioridad. Mahoney y Solomon (2016) quisieron examinar los efectos de la intervención en los estilos de interacción de los padres durante el compromiso social y determinar los cambios obtenidos en la sintomatología autista de los niños. 
Casenhiser y colaboradores (2011) desarrollan un ensayo clínico de una intervención basada en DIR $\odot$ en MEHRIT (Milton y Ethel Harris Research Initiative Tratament). El objetivo de esta intervención fue evaluar mejoras en las interacciones sociales en niños diagnosticados con autismo a partir de la participación en este programa. Se seleccionó una muestra de 51 niños del programa que cumplieran los criterios de selección (haber completado 12 meses de intervención MEHRIT o de intervención en servicios comunitarios). El diagnóstico fue confirmado por el equipo de intervención mediante ADOS y ADI (Autism Diagnostic Interview: Le Couteur et al., 1989). La edad de todos los niños estuvo comprendida entre los 2 años y los 4 años y 11 meses. Las familias seleccionadas debían disponer de tiempo para este estudio ( 2 horas/semana de terapia y 3 horas/día para interactuar con su hijo). Las familias fueron asignadas de forma aleatoria a dos grupos: experimental (Intervención MEHRIT) y control (Tratamiento Comunitario Convencional mientras esperaban intervención MEHRIT). Los terapeutas participantes recibieron formación reglada DIR $\odot$ por medio de ICDL. Para evaluar las interacciones entre padres e hijos se utilizó una versión modificada del CBRS (Child Behavior Rating Scale: Kim y Mahoney, 2004); para la evaluación del desarrollo del lenguaje se utilizaron PLS-IV (Preschool Language Scale IV: Zimmerman, Steiner y Pond, 2002) y el CASL (Comprenhensive Assessment of spoken Language: Carrow-Woolfolk, 1999). Se consideró además importante el cumplimiento de las instrucciones del programa y adherencia al mismo por lo que se desarrolló un cuestionario específico para este fin (MEHRIT Fidelity Scale). Los resultados en la CBRS demostraron mejoras significativas en las relaciones padres e hijos en el grupo que recibió la intervención MEHRIT en cuanto al área de lenguaje las pruebas CASL y PLS evidenciaron mejoras en ambos grupos, aunque estas fueron mayores en el grupo MEHRIT. Este estudio ofrece datos sólidos acerca de la intervención que permiten evidenciar la eficacia de esta. No obstante, es necesario también marcar algunas lagunas como, por ejemplo, que el grupo control recibió menos horas de tratamiento que el grupo MEHRIT. De la misma forma, los padres participantes en la intervención MEHRIT tuvieron mayor participación durante el proceso, lo cual no se realizó en el grupo control, aspectos que pudieron influir en los resultados finales.

\section{Valoración de las evidencias}

El auge del enfoque basado en la evidencia ha generado multitud de criterios para valorar el nivel de evidencia que aporta un resultado dado (Harris et al., 2001; Kemdall et al., 2013; Manglione et al., 2012; Reichow, Hume, Barton y Boyd, 2018). Para nuestros objetivos, destaca la iniciativa de la Journal of Clinical Child $y$ Adolescent Psychology (JCCAP) (desarrollada específicamente para evaluar estudios de tratamientos para trastornos de conducta infantil). Smith y Iadarola (2015) adaptan los criterios de la JCCAP al caso específico de los tratamientos en niños con TEA. A partir de este trabajo, se han desarrollado unos criterios de valoración propios. Los criterios JCCAP consideran que unos resultados pueden ser adecuados o tener una 
evidencia bien establecida cuando se dispone de varios ensayos clínicos aleatorios bien definidos metodológicamente. Los ensayos clínicos aleatorizados, tienen como objetivo reducir los sesgos experimentales al probar la efectividad de un método o tratamiento nuevo. Este tipo de estudios, generalmente comparativo entre dos métodos (convencional o control y experimental), son poco frecuentes por las dificultades intrínsecas que implican y por las limitaciones éticas que impiden en ocasiones la asignación aleatoria a un tipo u otro de intervención (Kendall, 2003). Sin embargo, aportan un valor incuestionable y son imprescindibles para el desarrollo de la práctica basada en la evidencia (Stang, 2011). La idea básica es que los sujetos son asignados a los grupos de intervención o control de forma aleatoria garantizando de esta manera que sean estadísticamente equivalentes. Pueden hacerse de forma abierta, dando a conocer cuál de los dos grupos será grupo experimental, o de forma ciega, cuando se asigna al grupo de forma que el participante no sabe si le darán tratamiento (intervención) o no (Bhide, Shah y Acharya, 2018). Uno de los problemas con los que se encuentra la investigación es la heterogeneidad de la población diagnosticada con TEA (Sherer y Schreibman, 2005), que obliga a tener grupos heterogéneos o tener que hacer una selección basada en perfiles neuropsicológicos complejos y difíciles de conseguir.

Los criterios que proponemos para este trabajo (ver Tablas 1 y 2 ) diferencian entre estudios con evidencias bien establecidas o adecuadas (Nivel 1), de los estudios con resultados probable o posiblemente eficaces (Nivel 2), en el tamaño de la muestra y número de estudios. Entre los primeros, a su vez, se diferencia entre métodos, que, debido al número de RCT publicados, se pueden analizar resultados estadísticos conjuntos o metaanálisis, de aquellos métodos que solo disponen de un RCT, pero este es muy robusto por disponer de una muestra estratificada amplia. Entre los de Nivel 2, también distingue entre estudios RCT con muestras pequeñas y metaanálisis de series de estudios de un solo caso.

Las evidencias de estudios cuasi-experimentales por definición (Nivel 3), son aquellos que aún no han alcanzado los niveles de evidencia adecuados, los resultados pueden apuntar hacia nuevos estudios en el futuro con dirección positiva. Se trata generalmente de estudios cuasi-experimentales en los que se toma medida de uno o dos grupos. Su característica fundamental es la simplicidad y economía en el desarrollo. Pueden agruparse en dos tipos, de un solo grupo con pretest y postest y de varios grupos, estos últimos se diferencian de los experimentales propiamente dichos en que los sujetos forman parte de grupos naturales no aleatorios. Este tipo de estudios no son concluyentes, pero pueden ser una herramienta poderosa, sobre todo cuando aún no son posibles experimentos aleatorizados. Permiten tener una visión general y hacer un seguimiento para determinar o confirmar las razones de los resultados encontrados (Morgan, Glinger y Harmon, 2000). También pueden considerarse incluidos en este nivel los estudios de casos. La investigación con casos únicos (ECU: Estudio de Caso Único) es experimental más que descriptiva o correlacional. Su objetivo es documentar las relaciones entre la variable independiente (tratamiento experimental) y dependientes. Al tratase de un caso único, las diferencias individuales que afectan a la validez interna de la experiencia quedan controladas. La acumulación de los resultados de 
diferentes ECU sobre una misma problemática y con una misma metodología puede incrementar la evidencia de esta (Horner et al., 2005), aumentando la validez externa. Por último, existen estudios (Nivel 4) que podríamos calificar de evidencias cuestionables debido a las características de la metodología utilizada.

\begin{tabular}{|l|l|l|l|}
\hline \multicolumn{3}{|c|}{ TABLA 1. Niveles de evidencia modificados a partir de los criterios } \\
originales del JCCAP
\end{tabular}

Dentro de cada nivel o categoría, a su vez, los estudios pueden clasificarse o valorarse en función de la calidad del estudio. Para ello, los criterios JCCAP incluyen varios apartados que aquí se resumen en uno específico de cinco puntos donde cada uno de ellos puede ser valorado de $\mathrm{O}$ a 3 puntos según el nivel de cumplimiento. 


\begin{tabular}{|l|l|l|}
\hline \multicolumn{3}{|c|}{ TABLA 2. Valoración de los criterios de calidad del estudio desde el punto } \\
de vista metodológico
\end{tabular}

Se han aplicado estos criterios a los 16 estudios anteriormente expuestos y los resultados se muestran en la Tabla 3. En general, como se puede observar, los estudios de caso único son mal valorados de la misma forma que los estudios cuasi-experimentales. En cuanto a los ensayos clínicos, podemos ver como los mejor evaluados son precisamente los estudios de Mahoney y Solomon (2016) y Casenhiser et al. (2011) que en contraposición no aplican estrictamente la metodología DIR/Floortime $\odot$, sino que desarrollan un programa propio basado en los principios DIR/ Floortime $\odot$. 
REVISIÓN DE EVIDENCIAS DE LAS TÉCNICAS DE DIR/FLOORTIME ${ }^{\text {TM }}$ PARA LA INTERVENCIÓN EN NIÑOS Y NIÑAS CON TRASTORNOS DEL ESPECTRO DEL AUTISMO

LILIANA ROJAS TORRES, YURENA ALONSO ESTEBAN Y FRANCISCO ALCANTUD-MARÍN

\begin{tabular}{|c|c|c|c|c|c|c|c|c|c|}
\hline \multirow{14}{*}{ 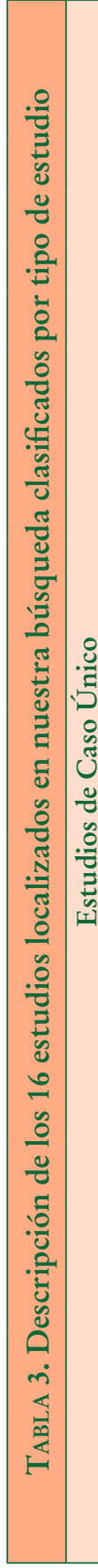 } & \multirow{6}{*}{ 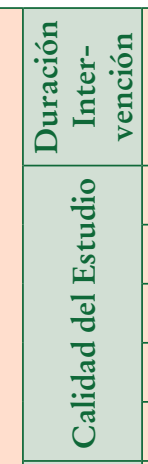 } & & & & 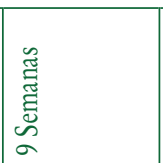 & 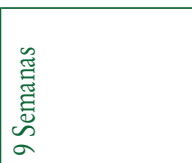 & 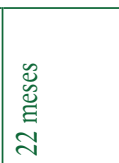 & 壱 & 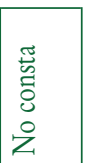 \\
\hline & & $\sum$ & 0 & - & 0 & $\sim$ & - & 0 & 0 \\
\hline & & $\sum_{\Sigma}^{\nabla}$ & - & $\sim$ & - & $\sim$ & $\sim$ & - & 0 \\
\hline & & $\sum_{\bar{\lambda}}^{m}$ & - & 0 & 0 & o & 0 & 0 & 0 \\
\hline & & $\tilde{\Sigma}$ & - & - & - & $\sim$ & $\sim$ & $\sim$ & 0 \\
\hline & & $\bar{\Sigma}$ & 0 & 0 & 0 & 0 & 0 & 0 & 0 \\
\hline & 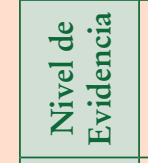 & & $\tilde{m}$ & $\stackrel{m}{m}$ & $\tilde{m}$ & $\stackrel{m}{m}$ & $\vec{m}$ & 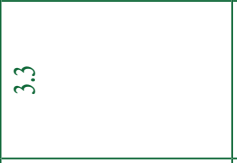 & $\stackrel{m}{m}$ \\
\hline & & 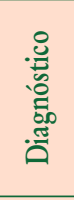 & 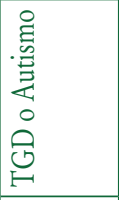 & 崶 & 葛 & 坒 & 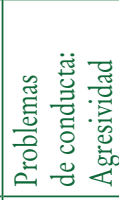 & 竞 & 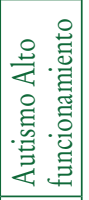 \\
\hline &.$\stackrel{\vec{J}}{\vec{B}}$ & 苞 & 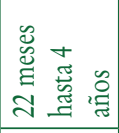 & 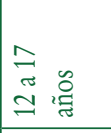 & 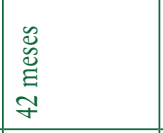 & 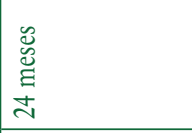 & 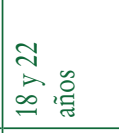 & 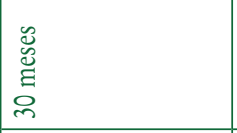 & 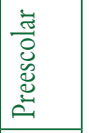 \\
\hline & & 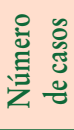 & 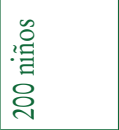 & 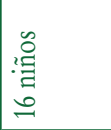 & 影 & 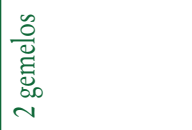 & 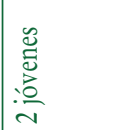 & 冒 & 总 \\
\hline & 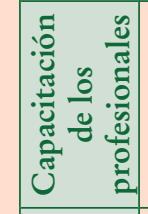 & & 总 & 总 & 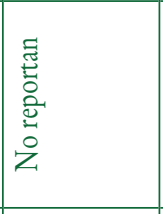 & 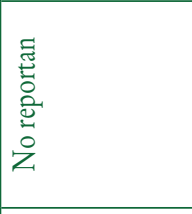 & 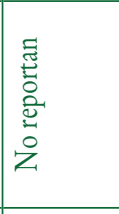 & 总 & 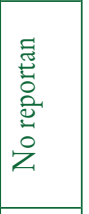 \\
\hline & 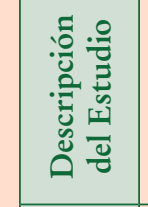 & & 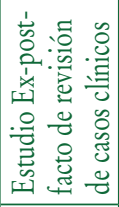 & 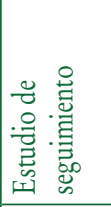 & 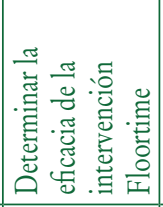 & 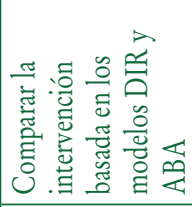 & 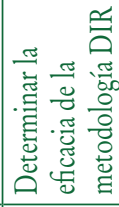 & 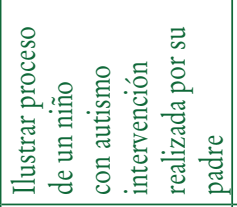 & 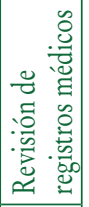 \\
\hline & $\stackrel{n}{\pi}$ & & 崩 & 虫 & $\mathbb{U}$ & 死 & 芯 & 萜 & o \\
\hline & 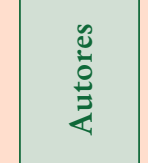 & & 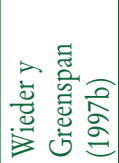 & 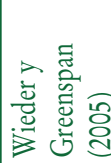 & 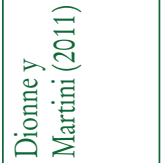 & 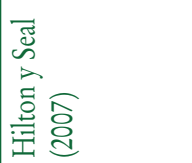 & .气 & 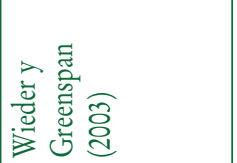 & 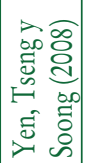 \\
\hline
\end{tabular}

Ediciones Universidad de Salamanca / CC BY-NC-ND

Siglo Cero, vol. 51 (2), 2020, abril-junio, pp. 7-32 
REVISIÓN DE EVIDENCIAS DE LAS TÉCNICAS DE DIR/FLOORTIME TM PARA LA INTERVENCIÓN EN NIÑOS Y NIÑAS CON TRASTORNOS DEL ESPECTRO DEL AUTISMO

LILIANA ROJAS TORRES, YURENA ALONSO ESTEBAN Y FRANCISCO ALCANTUD-MARÍN

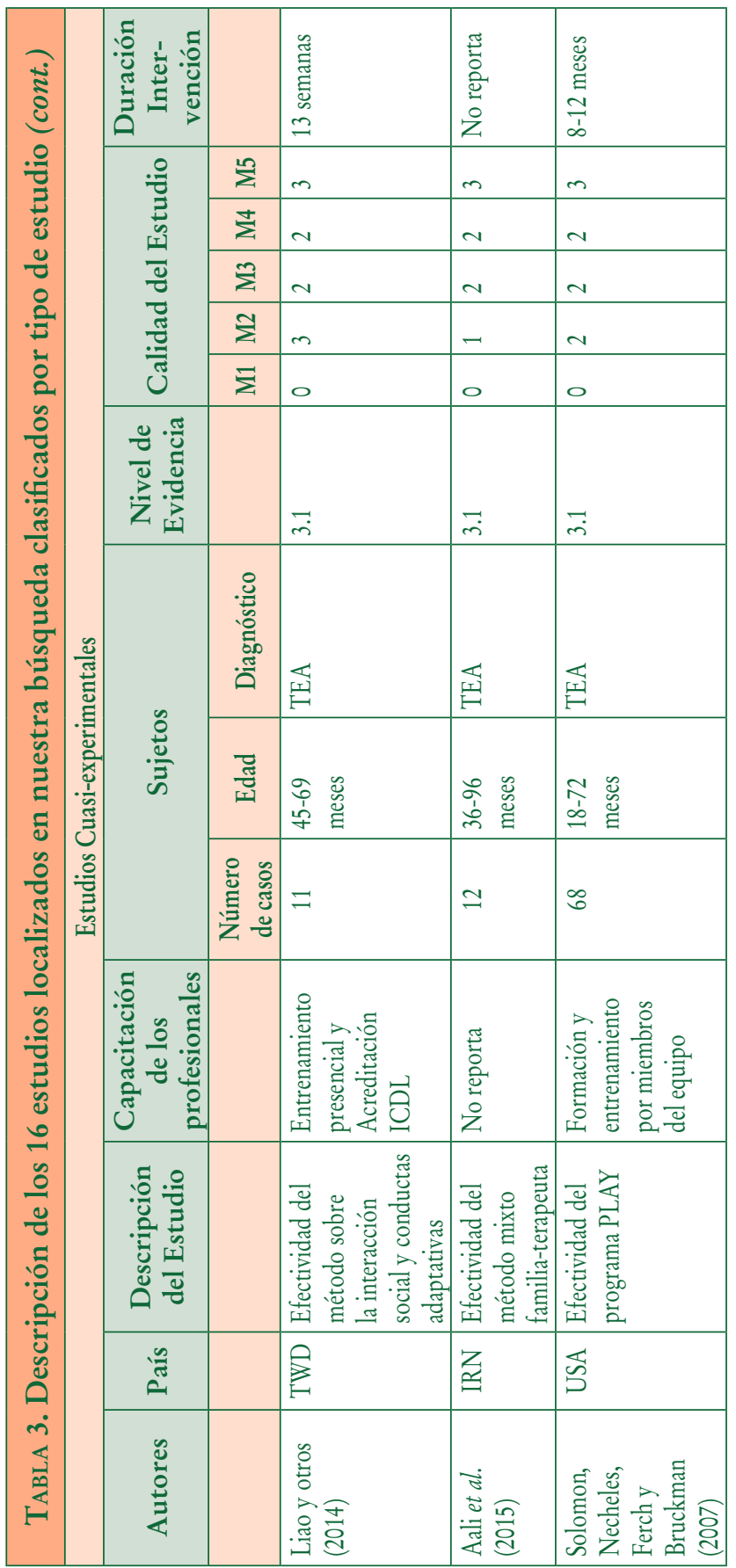

Ediciones Universidad de Salamanca / CC BY-NC-ND

Siglo Cero, vol. 51 (2), 2020, abril-junio, pp. 7-32 
REVISIÓN DE EVIDENCIAS DE LAS TÉCNICAS DE DIR/FLOORTIMETM PARA LA INTERVENCIÓN EN NIÑOS Y NIÑAS CON TRASTORNOS DEL ESPECTRO DEL AUTISMO

LILIANA ROJAS TORRES, YURENA ALONSO ESTEBAN Y FRANCISCO ALCANTUD-MARÍN

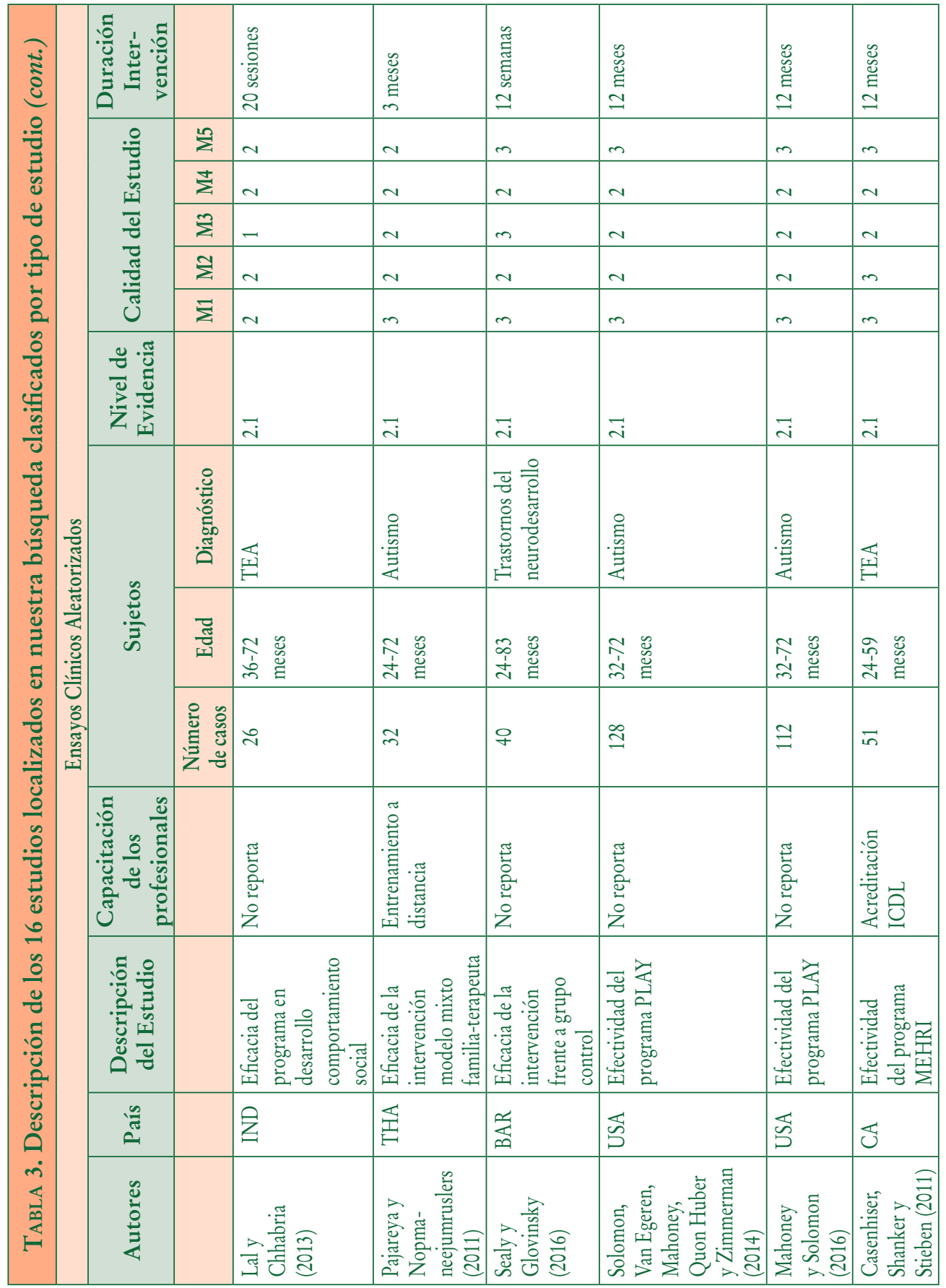

Ediciones Universidad de Salamanca / CC BY-NC-ND

Siglo Cero, vol. 51 (2), 2020, abril-junio, pp. 7-32 
REVISIÓN DE EVIDENCIAS DE LAS TÉCNICAS DE DIR/FLOORTIMETM PARA LA INTERVENCIÓN EN NIÑOS Y NIÑAS CON TRASTORNOS DEL ESPECTRO DEL AUTISMO

LILIANA ROJAS TORRES, YURENA ALONSO ESTEBAN Y FRANCISCO ALCANTUD-MARÍN

\section{Conclusiones}

Del análisis de los estudios aquí referenciados se desprende, en primer lugar, que el número de estudios empíricos sobre el DIR/Floortime ${ }^{\mathrm{TM}}$ es escaso. Se hace necesario un mayor número de RCT desarrollados por equipos independientes en diferentes lugares. Aunque hemos encontrado seis RCT, estos no tienen la suficiente homogeneidad como para poder someterlos a un metaanálisis. En algunos casos, como los estudios de Solomon et al. (2014) y Casenhiser et al. (2011), entendemos que no se ha aplicado estrictamente el modelo DIR/Flootime ${ }^{\mathrm{TM}}$, aunque sí las técnicas Floortime. En otros casos, la intervención ha pilotado sobre un modelo de intervención en el hogar (Pajareya y Nopmaneejumruslers, 2011) o se ha intervenido con el objetivo de mejorar aspectos de interacción comunicativa y en otros, con la finalidad de disminuir la agresividad y problemas de conducta. En cuanto a cómo se han medido los resultados, la heterogeneidad es también manifiesta. Lo más común es utilizar la escala FEAS (Greenspan y Degangi, 1992). Pero también se han desarrollado adaptaciones o pruebas ad hoc como BSFS (Lal y Chhabria, 2013). La edad de aplicación y el diagnóstico son otras tantas fuentes de variación. En alguno de los trabajos se informa de la combinación de dinámicas, rutinas y metodologías de otras intervenciones, ya que la gran mayoría de estos niños se encontraban inscritos en programas comunitarios o de otro tipo (terapia del lenguaje, ABA, etc.) para el fortalecimiento de diversas áreas del desarrollo (Casenhiser, Shanker y Stieben, 2011; Hilton y Seal, 2007; Pajareya y Nopmaneejumruslers, 2011; Pajareya y Nopmaneejumruslers, 2012), razón por la cual no se podría decir que el modelo DIR @ per se es el que logra los avances en el niño. Teniendo en cuenta que las principales características en los TEA son las alteraciones en la interacción social, la comunicación y la conducta e inflexibilidad de intereses (Tobón, 2012), podemos decir que el método DIR/Floortime ${ }^{\mathrm{TM}}$ es prometedor, aunque las evidencias aún no lo demuestren con rotundidad.

Por otro lado, uno de los focos más importantes en el tratamiento de los niños con autismo es la interacción social. En la presente revisión se encontró que la implementación de la metodología Floortime ${ }^{\mathrm{TM}}$ parece fortalecer la reciprocidad social; Casenhiser, Shanker y Stieben, 2011; Hilton y Seal, 2007; Lal y Chhabria, 2013; Mahoney y Solomon, 2016; Pajareya y Nopmaneejumruslers, 2011; Pajareya y Nopmaneejumruslers, 2012; Solomon, Van Egeren, Mahoney, Huber y Zimmerman, 2014, demuestran las ganancias que tienen los niños con TEA en el funcionamiento social, logrando mejoras significativas en el desarrollo funcional emocional, establecimiento de vínculos sociales, capacidad de relacionarse e involucrarse y comunicarse de manera positiva con otras personas de su entorno.

Finalmente, se encontró que la intervención basada en DIR/Floortime ${ }^{\mathrm{TM}}$ permite que los niños con TEA logren mejoras en la severidad de su sintomatología, así como ganancias en el desarrollo funcional, Aali, Yazdi, Abdekhodaei, Chamanabad y Moharreri, 2015; Mahoney y Solomon, 2016; Pajareya y Nopmaneejumruslers, 2011; Pajareya y Nopmaneejumruslers, 2012; Solomon, Necheles, Ferch y Bruckman, 2007; Solomon, Van Egeren, Mahoney, Huber y Zimmerman, 2014, informan de ganancias en el desarrollo de los niños, reduciendo la gravedad en la sintomatología autista. 
Hacen falta más estudios RCT que sigan rigurosamente la metodología DIR/ Floortime $^{\mathrm{TM}}$, en niños y niñas con TEA de edades similares (24-48 meses) y con los mismos niveles de gravedad, los cuales sean desarrollados por equipos independientes, pero con formación acreditada, con control del seguimiento y con instrumentos psicométricos debidamente validados para poder llegar a un metaanálisis que arroje evidencias irrefutables sobre los resultados de esta técnica.

\section{Referencias bibliográficas}

Aali, S., Yazdi, S., Abdekhodaei, M., Chamanabad, A. y Moharreri, F. (2015). Developing a mixed family-focused therapy based on integrated human developmental model and comparing its effectiveness with Floortime play-therapy on the developmental family function and the functional-emotional development of children with autism. Journal of Fundamental of Mental Health, 87-97.

Aber, J., Slade, A., Berger, B., Bresgi, I. y Kaplan, M. (1985). The parent development interview. New York: Barnard College Departament of Psychology.

Abidin, R. (1990). Parenting stress index. Odessa, Florida: Psychological Assessment Resources. APA (1994). Diagnostic and statistical manual of mental disorders. 4th Edition. Washington: American Psychiatric Association.

APA (2013). Diagnostic and statistical manual of mental disorders. 5th Edition. Arlington: American Psychiatric Publishing.

Artigas-Pallarés, J. (2007). Detección precoz de los trastornos del neurodesarrollo. Atención precoz de los trastornos del neurodesarrollo. A favor de la intervención precoz de los trastornos del neurodesarrollo. Revista de Neurología, 44(Supl. 3), s31-S34. Recuperado el 5 de abril de 2015, de http://www.neurologia.com/pdf/Web/44S03/xS03S031.pdf.

Baio, J., Wiggins, L., Chistensen, D., Maenner, M., Daniels, J., Warren, Z., ... Dowling, N. (2018). Prevalence of autism spectrum disorder among children aged 8 years - autism and developmental disabilities monitoring network, 11 Sites, United States, 2014. MMWR Morbility and Mortality Weekly Report, 67(6), 1-23. Recuperado el 2 de may de 2018, de https://www.cdc.gov/mmwr/volumes/67/ss/ss6706a1.htm?s_cid=ss6706a1_w.

Bhide, A., Shah, P. y Acharya, G. (2018). A simplified guide to randomized controlled trials. Acta Obstetrica et Gynecologica Scandinavica, 97(4), 380-387. https://doi.org/10.1111/aogs.13309.

Bratton, S., Ray, D., Rhine, T. y Jones, L. (2005). The efficacy of play therapy with children: a meta-analytic review of treatment outcomes. Professional Psychology, Research and Practice, 36(4), 376-390. https://doi.org/10.1037/0735-7028.36.4.376.

Carrow-Woolfolk, E. (1999). Comprehensive assessment of spoken language. Circle Pines, MN: American Guidance Service.

Casenhiser, D., Shanker, S. y Stieben, J. (2011). Learning through interaction in children with autism: preliminary data from a social-communication-based intervention. Autism, 220-241. https://doi.org/10.1177/1362361311422052.

Case-Smith, J. y Arbesman, M. (2008). Evidence-based review of interventions for autism used in or of relevance to occupational therapy. American Journal of Occupational Therapy, 62, 416-429.

Christian, G. (2011). A person-centered approach to problem behavior: using DIR/Floortime with adults who have severe developmental delays. The NADD Bulletin, 14(2), 21-31. Recuperado el 10 de noviembre de 2018, de http://thenadd.org/nadd-bulletin/. 
REVISIÓN DE EVIDENCIAS DE LAS TÉCNICAS DE DIR/FLOORTIME TM PARA LA INTERVENCIÓN EN NIÑOS Y NIÑAS CON TRASTORNOS DEL ESPECTRO DEL AUTISMO

LILIANA ROJAS TORRES, YURENA ALONSO ESTEBAN Y FRANCISCO ALCANTUD-MARÍN

CRD (2008). Systematic reviews. CRD's guidance for undertaling reviews in bealth care. York: University of York. Recuperado el 10 de april de 2014, de http://www.york.ac.uk/inst/crd/ pdf/Systematic_Reviews.pdf.

Dawson, G., Jones, E., Merkle, K., Venema, K., Lowy, R., Faja, S., ... Webb, S. (2012). Early behavioral intervention is associated with normalized brain activity in young children with autism. Journal of the American Academy of Child and Adolescent Psychiatry, 51(11), 11501159. https://doi.org/10.1016/j.jaac.2012.08.018.

Dionne, M. y Martini, R. (2011). Floor Time Play with a child with autism: a single-subject study. Canadian Journal of Occupational Therapy, 196-203. https://doi.org/10.2182/ cjot.2011.78.3.

Dunn, W. (1999). The sensory profile. San Antonio. Texas: Psycgilogical Corporation.

Eldevik, S., Hasting, R., Hughes, J., Jahr, E., Eikeseth, S. y Cross, S. (2009). Meta-analysis of early intensive behavioral intervention for children with autism. Journal of Clinical Child and Adolescent Psychology, 38(3), 439-450. https://doi.org/10.1080/15374410902851739.

Eyberg, S. (1988). Parent-child interaction therapy: integration of traditional and behavior concerns. Child and Family Behavior Therapy, 10(1), 33-46. https://doi.org/10.1300/ J019v10n01_04.

Fein, D., Barton, M., Eigsti, I., Kelley, E., Naigles, L., Schultz, R., ... Tyson, K. (2013). Optimal outcome in individuals with a history of autism. Journal of Child Psychology and Psychiatry, 54(2), 195-205.

Fuentes-Biggi, J., Ferrari-Arroyo, M., Boada-Muñoz, L., Touriño-Aguilera, E., Artigas-Pallarés, J., Belinchón-Carmona, M., ... Posada-de La Paz, M. (2006). Guía de buenas prácticas para el tratamiento de los trastornos del espectro autista. Revista de Neurología, 43(7), 425-438. https://doi.org/10.33588/rn.4307.2005750.

Garner, P., Jones, D. y Miner, J. (1994). Social competence among low-income preschoolers: emotion socialization practices and social cognitive correltaes. Child Development, 65(2), 622-637. https://doi.org/10.2307/1131405.

GinotT, H. (1959). The theory and practice of therapeutic intervention in child treatment. Journal of Consulting and Clinical Psychology, 23(2), 160-166. https://doi.org/10.1037/ h0046805.

GinotT, H. (1960). A rationale for selecting toys in play therapy. Journal of Consulting and Clinical Psychology, 24(3), 243-246. https://doi.org/10.1037/h0043980.

Greenspan, J. y Greenspan, S. (2002). Functional Emotional Developmental Questionnaire (FEDQ) for childhood: a preliminary report on the questions and their clinical meaning. The Journal of Developmental and Learning Disorders, 6, 71-116. Recuperado el 3 de septiembre de 2019, de http://www.centerforthedevelopingmind.com/sites/default/files/ developmental-model-for-research.pdf.

GrEENSPAN, S. (1992). Infancy and early childhood: the practice of clinical assessment and intervention with emotional and developmental challenges. Madison: International Universities Press.

Greenspan, S. y DeGangi, G. (2001). Research on the FEAS: test development, reliability, and validity studies. En S. Greenspan, G. DeGangi y S. Wieder, The Functional Emotional Assessment Scale (FEAS) for infancy and early childhood. Clinical and research applications (pp. 167-247). Bethesda, MD: The Interdisciplinary Council on Development and Leatning, Inc. Recuperado el 28 de agosto de 2019, de http://www.icdl.com/research/ functional-emotional-assessment-scale.

Greenspan, S. y Wieder, S. (1997a). An integrated developmental approach for young children with severe difficulties in relating and communicating. En S. GreEnspan, B. Kalmanson, 
REVISIÓN DE EVIDENCIAS DE LAS TÉCNICAS DE DIR/FLOORTIMETM PARA LA INTERVENCIÓN EN NIÑOS Y NIÑAS CON TRASTORNOS DEL ESPECTRO DEL AUTISMO

LILIANA ROJAS TORRES, YURENA ALONSO ESTEBAN Y FRANCISCO ALCANTUD-MARÍN

R. Shahmoon-Shanok, G. Wieder, G. Willamson y M. Anzalone, Assessing and treating infants and young children with severe difficulties in relaring and communicating (pp. 5-18). Washington: Zero to Three.

Greenspan, S. y Wieder, S. (1997b). Developmental patterns and outcomes in infants and children with disorders in relating and communicating: a chart review of 200 cases of children with autistic spectrum diagnoses. The Journal of Developmental and Learning Disorders, 11-38.

Greenspan, S. y Wieder, S. (1998). The child with special needs. Encouraging intellectual and emotional growth. Massachusetts: Perseus Publishing.

Greenspan, S. y Wieder, S. (1999). A functional developmental approach to autism spectrum disorders. Research and Practice for Persons with Severe Disabilities, 24(3), 147-161. https://doi.org/10.2511/rpsd.24.3.147.

Greenspan, S. y Wieder, S. (2006). Engaging autism: using the Floortime approach to belp children relate, communicate, and think. Cambridge, MA: SaCapo Press.

Harris, R., Helfand, M., Woolf, S., Lohr, K., Mulrow, C., Teutsch, S. y Atkins, D. (2001). Current methods of the US Preventive Services Task Force: a review of the process. American Journal of Preventive Medicine, 20(3), 21-35. https://doi.org/10.1016/S07493797(01)00261-6.

Hillman, H. (2018). Child-centered play therapy as an intervention for children with autism: a literature review. International Journal of Play Therapy, 27(4), 198-204. https://doi. org/10.1037/pla0000083.

Hilton, J. y SeAL, B. (2007). Brief report: comparative ABA and DIR trials in twin brothers with autism. Journal Autism Developmental Disorders, 1197-1201. https://doi.org/10.1007/ s10803-006-0258-z.

Horner, R., Carr, E., Halle, J., McGee, G., Odom, S. y Wolery, M. (2005). The use of single-subject research to identify evidence-based practice in special education. Exceptional Children, 71(2), 165-179. https://doi.org/10.1177/001440290507100203.

IECS (2007). Terapia de juego en el piso para el autismo. Buenos Aires, Argentina: Institute for Clinical Efectiveness and Health Policy. Recuperado el 23 de junio de 2017, de https:// www.iecs.org.ar/publicacion/? id=4350.

Kasari, C., Gulsrud, A., Freeman, S., Paparella, T. y Hellemann, G. (2012). Longitudinal follow-up of children with autism receiving targed interventions on joint attention and play. Journal of the American Academy of Child and Adolescent Psychiatry, 51(5), 487-495. https://doi.org/10.1016/j.jaac.2012.02.019.

Kemdall, T., Megnin-Viggars, O., Gould, N., Taylor, C., Burt, L. y Baird, G. (2013). Management of autism in children and young people: summary of NICE and SCIE guidance. British Medical Journal, 347, f4865. https://doi.org/10.1136/bmj.f4865.

Kendall, J. (2003). Designing a research project: randomised controlled trials and their principles. Emergency Medicine Journal, 20(2), 164-168. https://doi.org/10.1136/emj.20.2.164.

Kim, J. y Mahoney, G. (2004). The effects of mother's style of interaction on children's engagement: implications for using responsive interventions with parents. Topics in Early Childhood Special Education, 24(1), 31-38. https://doi.org/10.1177/02711214040240010301.

LAL, R. y ChHAbria, R. (2013). Early intervention of autism: a case for Floor Time approach. Recent Advances in Autism Spectrum Disorders. https://doi.org/10.5772/54378.

LANG, R., Hancock, T. y Singh, N. (2016). Overview of early intensive behavioral intervention for children with autism. En R. LANG, T. Hancock y N. Singh, Early intervention for young children with autism spectrum disorder (pp. 1-14). Basel, Switzerland: Springer 
REVISIÓN DE EVIDENCIAS DE LAS TÉCNICAS DE DIR/FLOORTIME TM PARA LA INTERVENCIÓN EN NIÑOS Y NIÑAS CON TRASTORNOS DEL ESPECTRO DEL AUTISMO

LILIANA ROJAS TORRES, YURENA ALONSO ESTEBAN Y FRANCISCO ALCANTUD-MARÍN

International Publishing. Recuperado el 10 de junio de 2017, de https://link.springer.com/ chapter/10.1007/978-3-319-30925-5_1.

Le Couteur, A., Rutter, M., Lord, C., Rios, P., Robertson, S., Holdgrafer, M. y McLennan, J. (1989). Autism diagnostic interview: a semistructured interview for parents and caregivers of autistic persons. Journal of Autism and Developmental Disorders, 19, 363 387. https://doi.org/10.1007/BF02212936.

Leblanc, M. y Ritchie, M. (2001). A meta-analysis of play therapy outcomes. Counselling Psychology Quarterly, 14(2), 149-163. https://doi.org/10.1080/09515070110059142.

Liao, S., Hwang, Y., Chen, Y., Lee, P., Chen, S. y Lin, L. (2014). Home-based DIR/Floortime $^{\mathrm{TM}}$ intervention program for preschool children with autism spectrum disorders: preliminary findings. Physical and Occupational Therapy in Pediatrics, 34(4), 356-367. https:// doi.org/10.3109/01942638.2014.918074.

Lord, C., Rutter, M. y Le Couteur, A. (1995). Autism Diagnostic Observation Schedule (ADOS) Manual. Los Angeles CA: Western Psychological Services.

LovaAs, I. (1987). Behavioral treatment and normal educational and intellectual functioning in young autistic children. Journal of Consulting and Clinical Psychology, 55, 3-9.

Mahoney, G. y Solomon, R. (2016). Mechanism of developmental change in the PLAY Project Home Consultation Program: evidence from a randomized control trial. Journal Developmental Disorders, 1860-1871. https://doi.org/10.1007/s10803-016-2720-x.

Makrygiam, M. y Reed, P. (2010). A meta-analytic review of the effectiveness of behavioural early intervention programs for children with autistic spectrum disorders. Research in $\mathrm{Au}$ tism Spectrum Disroders, 4, 577-593. https://doi.org/10.1016/j.rasd.2010.01.014.

Makrygianni, M. y Reed, P. (2010). A meta-analytic review of the effectiveness of behavioural early intervention programs for children with autistic spectrum disorders. Research in Autism Spectrum Disorders, 4(4), 577-593. https://doi.org/10.1016/j.rasd.2010.01.014.

Manglione, M., Gans, D., Das, L., Timbie, J., Kasari, C., Technical Expert Panel y Hrsa Airb Network (2012). Nonmedical interventions for children with ASD: recommended guidelines and further research needs. Pediatrics, 130, S169. https://doi.org/10.1542/ peds.2012-09000.

Mastrangelo, S. (2009). Play and the child with autism spectrum disorder: from possibilities to practice. International Journal of Play Therapy, 18(1),13-30. https://doi.org/10.1037/a0013810.

Moher, D., Liberati, A., Tetzlaff, J. y Altman, D. (2009). Preferred reporting items for systematic reviews and meta-analyses: the PRISMA statement. PLoS Medicine, 6(7), e1000097. https://doi.org/10.1371/journal.pmed.1000097.

Morgan, G., Glinger, J. y Harmon, R. (2000). Quasi-experimental designs. Journal of the American Academy of Child and Adolescent Psychiatry, 39(6), 794-796. https://doi. org/10.1097/00004583-200006000-00020.

Pajareya, K. y Nopmaneejumruslers, K. (2011). A pilot randomized controlled trial of DIR/Floortime ${ }^{\mathrm{TM}}$ parent training intervention for pre-school children with autistic spectrum disorders. Autism, 563-577. https://doi.org/10.1177/1362361310386502.

Pajareya, K. y Nopmaneejumruslers, K. (2012). A one-year prospective follow-up stu$\mathrm{dy}$ of a DIR/Floortime ${ }^{\mathrm{TM}}$ parent training intervention for pre-school children with autistic spectrum disorders. Journal Med Association Thai, 1184-1193. https://doi. org/10.1177/1362361310386502.

PDM TAsK Force (2006). Psychodynamic diagnostic manual (PDM). Silver Spring, MD: Aliance of Psychoanalytic Organizations.

Piaget, J. (1952). Play, dreams and imitation in childhood [La formación del símbolo en el niño: imitación, juego y sueños. New York, US: W W Norton \& Co.

Ediciones Universidad de Salamanca / CC BY-NC-ND

Siglo Cero, vol. 51 (2), 2020, abril-junio, pp. 7-32

$$
-30 \text { - }
$$


Prizant, B. y Wetherby, A. (1998). Understanding the continuum of discrete-trial traditional behavioral to social-pragmatic developmental approaches in communication enhancement for young children with autism/PDD. Seminars in Speech and Language, 19(4), 329-352. https://doi.org/10.1055/s-2008-1064053.

Reichow, B. (2012). Overview of meta-analyses on early intensive behavioral intervention for young children with autism spectrum disorders. Journal of Autism and Developmental Disorders, 42(4), 512-520. https://doi.org/10.1007/s10803-011-1218-9.

Reichow, B., Barton, E., Boyd, B. y Hume, K. (2014). Early Intensive Behavioral Intervention (EIBI) for young children with autism spectrum disorders: a systematic review. Campbell Systematic Reviews, 9. https://doi.org/10.4073/csr.2014.9.

Reichow, B., Hume, K., Barton, E. y Boyd, B. (2018). Early intensive behavioral intervention (EIBI) for young children with autism spectrum disorders (ASD). Cochrane Database of Systematic Reviews, 5, Art. No CD009260. https://doi.org/10.1002/14651858. CD009260.pub3.

Rogers, S., Estes, A., Lord, C., Vismara, L., Winter, J., Fitzpatrick, A., ... Dawson, G. (2012). Effects of a Brief Early Start Denver Model (ESDM)-based parent intervention on toddlers at risk for autism spectrum disorders: a randomized controlled trial. Journal of the American Academy of Child and Adolescent Psychiatry, 1052-1065.

Rogers, S., Estes, A., Vismara, L., Munson, J., Zierhut, C., Greenson, J., ... Talbott, M. (2018). Enhancing low-intensity coaching in parent implemented early Start Denver Model Intervention for Early Autism: a randomized comparison treatment trial. Journal of Autism and Developmental Disorders. https://doi.org/10.1007/s10803-018-3740-5.

Rogers, S. y Vismara, L. (2008). Evidence-based comprehensive treatments for early autism. Journal of Clinical Child and Adolescent Psychology, 37(1), 8-38. https://doi. org/10.1080/15374410701817808.

Rogers, S., Vismara, L., Wagner, A., McCormick, C., Young, G. y Ozonoff, S. (2014). Autism treatment in the first year of life: of infant start, a parent-implemented intervention for symptomatic infants. Journal of Developmental Disorders, 2981-2995.

Rutter, M., Bailey, A. y Lord, C. (2003). SCQ The Social Communication Questionnaire. Manual. Los Angeles, CA.: Western Psychological Services.

Schopler, E., Reichler, R. y Renner, B. (1986). The Childhood Autism Rating Scale (CARS): for diagnostic screening and classification of autism. New York: Irvington.

SEAly, J. y Glovinsky, I. (2016). Strengthening the reflective functioning capacities of parents who have a child with a neurodevelopmental disability through a brief, relationship focused intervention. Infant Mental Health Journal, 37(2), 115-124. https://doi.org/10.1002/ imhj.21557.

Sherer, M. y Schreibman, L. (2005). Individual behavioral profiles and predictors of treatment effectiveness for children with autism. Journal of Consulting and Clinical Psychology, 73(3), 525-538. https://doi.org/10.1037/0022-006X.73.3.525.

Simith, T. y Iadarola, S. (2015). Evidence base update for autism spectrum disorder. Journal of Clinical Child and Adolescent Psychology, 44(6), 897-922. https://doi.org/10.1080/15374 416.2015.1077448.

Solomon, R., Necheles, J., Ferch, C. y Bruckman, D. (2007). Pilot study of a parent training program for young children with autism: the Play Project Home Consultation program. Autism, 11(3), 205-224. https://doi.org/10.1177/1362361307076842.

Solomon, R., Van Egeren, L., Mahoney, G., Huber, M. y Zimmerman, P. (2014). PlAY Project Home Consultation Intervention Program for young children with autism spectrum disorders: a randomized controlled trial. Journal of Developmental and Behavioral Pediatrics, 475. https://doi.org/10.1097/DBP.0000000000000096. 
Sparrow, S., Cicchetti, D. y Balla, D. (2005). Vineland Adaptative Behaviro Scales. 2nd edition (VABS-II). New York: Pearson Inc.

STANG, A. (2011). Randomized controlled trials - an indispensible part of clinical research. Deutsches Ärzteblatt International, 108(39), 661-662. https://doi.org/10.3238/arztebl.2011.0661.

Товón, M. (2012). Detección temprana de autismo ¿es posible y necesaria? Revista CES Psicología. Recuperado el 10 de febrero de 2017, de https://dialnet.unirioja.es/servlet/ articulo? codigo $=3977325$.

Vivanti, G., Paynter, J., Ducan, E., Fothergill, H., Dissanayake, C., Rogers, S. y ASELCC Team. (2014). Effectiveness and feasibility of the Early Start Denver Model implemented in a group-based community childcare setting. Journal of Autism Developmental Disorders, 3140-3153. https://doi.org/10.1007/s10803-014-2168-9.

Warren, Z., McPheeters, M., Sathe, N., Foss-Feig, J., Glasser, A. y Veenstra-VanDERWEELE, J. (2011). A systematic review of early intensive intervention for autism spectrum disorders. Pediatrics, 127, 1098-4275, e1303-e1311. https://doi.org/10.1542/peds.2011-0426.

Wetherby, A. y Prizant, B. (1999). Communication and Symbolic Behavior Scales Manual. Chicago Illinois: Applied Symbolix, Inc.

Wieder, S. y Greenspan, S. (2003). Climbing the symbolic ladder in the DIR Model through Floor Time/Interactive Play. Autism, 7(4), 425-435. https://doi.org/10.1177/13623613030 07004008.

Wieder, S. y Greenspan, S. (2005). Can children with autism master the core deficits and become empathetic, creative, and reflective? The Journal of Developmental and Learning Disorders, 9, 39-61. Recuperado el 7 de septiembre de 2019, de https://pdfs.semanticscholar.org/1c2e/3fc7694a3448e569d52d3f40c86023603589.pdf.

Yen, H., Tseng, C. y Soong, W. (2008). A treatment based on the developmental individual difference, relationship-based model for two preschool children with high-functioning autism: a preliminary report (in Chinese). Formosa Journal of Mental Health, 21, 221-244.

Zimmerman, I., Steiner, V. y Pond, R. (2002). Preschool Language Scale (4th ed.). San Antonio TX: The Psychological Corporation. 